\title{
Changes in Cellular Properties during the Long-term Serial Culture of Lung Cells of Newborn Hamster and the Transformation into A Cells*
}

\author{
Yutaka Matsuya and Isao Yamane \\ Microbiology Division, The Research Institute for Tuberculosis, \\ Leprosy and Cancer, Tohoku University, Sendai
}

\begin{abstract}
Matsuya, Y. and Yamane, I. Changes in Cellular Properties during the Lony-term Serial Culture of Lung Cells of Newborn Hamster and the Transformation into $A$ Cells. Tohoku J. exp. Med., 1970, 102(1), 37-49. — - In the course of cell establishment, neonatal hamster lung cells progressively acquired various new properties and they showed morphological and karyological changes. The cells became capable of growing infinitely in albumin-free medium and these cells also had an ability to grow at low inoculation densities. The neoplastic property of the cells developed more slowly than their chromosomal alteration into the heteroploid range. The established line could similarly propagate in both the standard medium and serum albumin medium, and consequently the growth promotion by bovine albumin became almost negligible. - hamster cell; neoplastic property; chromosomal alteration
\end{abstract}

The serial culture method of lung cells of newborn hamster was already established and almost all cells evolved into established lines when the culture method $^{1,2}$ was followed. During the cell establishment, the cells acquired various new properties, which were different from those of primary cultures. ${ }^{3}$

The present communication describes the biological properties of lung cells of newborn hamster ('A' cells) which appeared in the course of long term serial culture employing a serum albumin medium. The transition of cell properties was associated with morphological and karyological changes of the cells.

\section{Methods}

Culture procedure. The culture method was essentially the same as those of the previous reports.1,2 Only massive cultures of ' $A$ ' cells were continuously obtained in square culture bottles $\left(2.5 \times 4.5 \times 8.0 \mathrm{~cm}^{3}\right)$ without $\mathrm{CO}_{2}$ gas supplement. The cells were plated with an inoculum density of $5 \times 10^{5}$ cells per bottle in $5 \mathrm{ml}$ of the medium and transferred every three or four days.

Transplantability. For the transplantation test, approximately 40-day-old Syrian hasmters were employed. The test was performed with a $5 \times 10^{5}$ cell suspension, which had been prepared by the use of ' $A$ ' cells at the 90 th cell generations or the 70 transfers and the cells were injected subcutaneously to the hamster's abdomen.

Received for publication, April 18, 1970.

* The third report of Culture of Syrian Hamster Fibroblasts in Albumin Fortified Medium. 
Cell morphology. In order to arrest the cell division in the metaphase, the cells were treated with $10^{-6} \mathrm{M}$ colchicine for ten hours. The hypotonic treatment was performed employing $1 / 3$ to $1 / 4$ strength of Hanks' solution. Chromosome flattening was made with the air drying method ${ }^{4}$ and the cells were stained with Giemsa solution.

\section{Results}

1) Emergence of an ability to sustain infinite propagation of cells in the standard medium

The growth rate of the cells from the primary culture rapidly fell in the course of serial transfers in the standard medium (ST medium) and the growth ceased by the 10th transfer. On the other hand, the cells carried in serum albumin fortified medium (BSA medium) showed a rapid and constant growth after they passed through a lag phase. ${ }^{1,2}$ The cells kept in BSA medium could continue to stand the serial transfers even in ST medium at the 14th cell generation or at the 23th transfer after they acquired an ability of constant growth in BSA medium as shown in Fig. 1. Finally, the cells were kept well in ST medium until the experiment was finished. Therefore, it may be concluded that the cells acquired a new property to sustain a constant stable growth, regardless of the presence or absence of albumin in the medium, and consequently the cells could dispense with albumin in continuous serial culture.

The growth potential of the cells in both ST and BSA media was compared

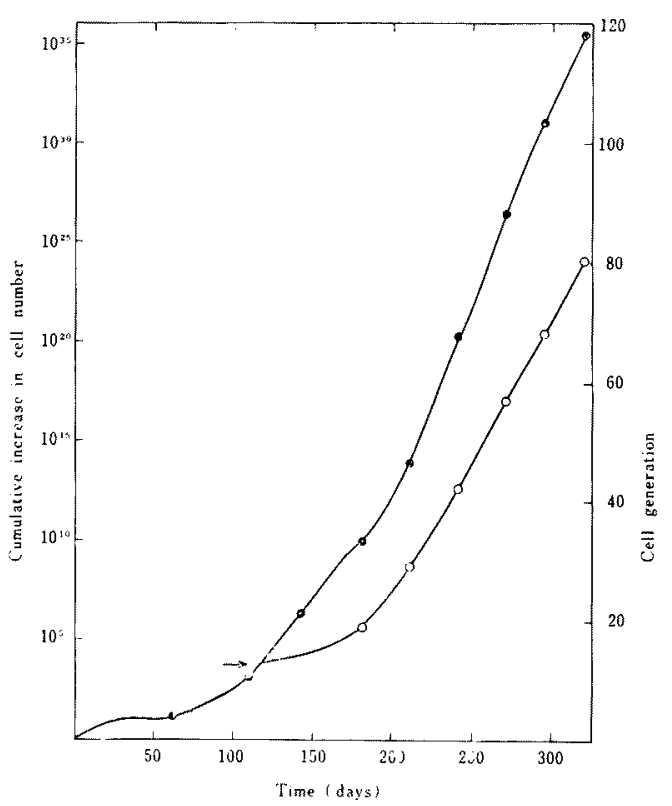

Fig. 1. Growth of 'A' cells carried in both BSA medium and ST medium for the long term. - ST medium. - - BSA medium. Arrow indicates the start of subculture in ST medium of the cells grown on BSA medium. Each point represents the cumulative cellular increase at every ten transfers. 


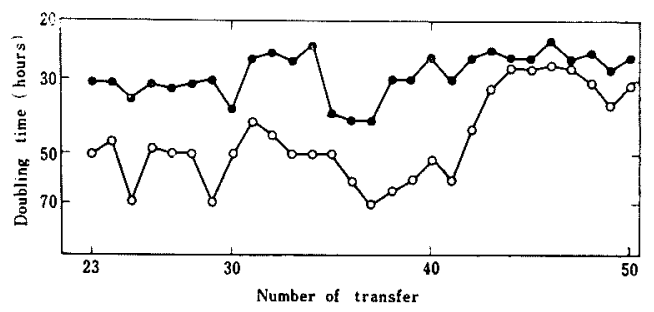

Fig. 2. Growth rate of 'A' cells in both BSA medium and ST medium at low inoculation density after establishment. @- ST medium. •- BSA medium.

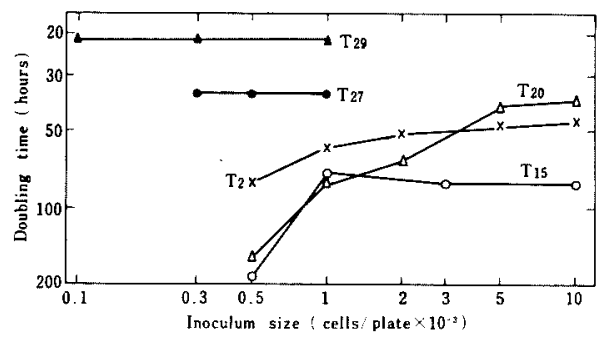

Fig. 3. Relation between the inoculation density and the growth rate of ' $A$ ' cells grown in BSA medium at different time. T number: Number of transfer.

with time spent in culture after the cells in massive cultures had acquired an ability to propagate infinitely in ST medium. The seed cultures kept in BSA medium were inoculated with $10^{4}$ cells per plate in $5 \mathrm{ml}$ of the media and incubated in both media for 7 days without changing the medium. The doubling times between the 23rd and 50th transfers in both media were plotted in Fig. 2. The cells in BSA medium could grow with a doubling time between 25 and 40 hours until the 40th transfer and thereafter the time was shortened to fall between 25 and 30 hours.

The cells carried in BSA medium were simultaneously inoculated into ST medium at the every transfer as shown in Fig. 2. The doubling time of the cells was between 40 and 70 hours until the 42 nd transfer, but the growth rate began to rise from the next transfer. The doubling time in ST medium, however, was never shortened below that in BSA medium throughout the present experiment. Therefore, it may be assumed that serum albumin had still an accessory growth promoting effect on the cells even after the cell establishment.

\section{2) Emergence of an ability to grow at low inoculation densities}

Todaro and Green ${ }^{5}$ advocated that one of the cellular properties which emerge very soon after the cell establishment is an ability to grow at low inoculation densities. Employing various cultures, the time was assessed when the cells acquired a capacity to grow at low inoculation densities. Fig. 3 shows a representative experiment of 'A' cells kept in BSA medium. The cells in the 


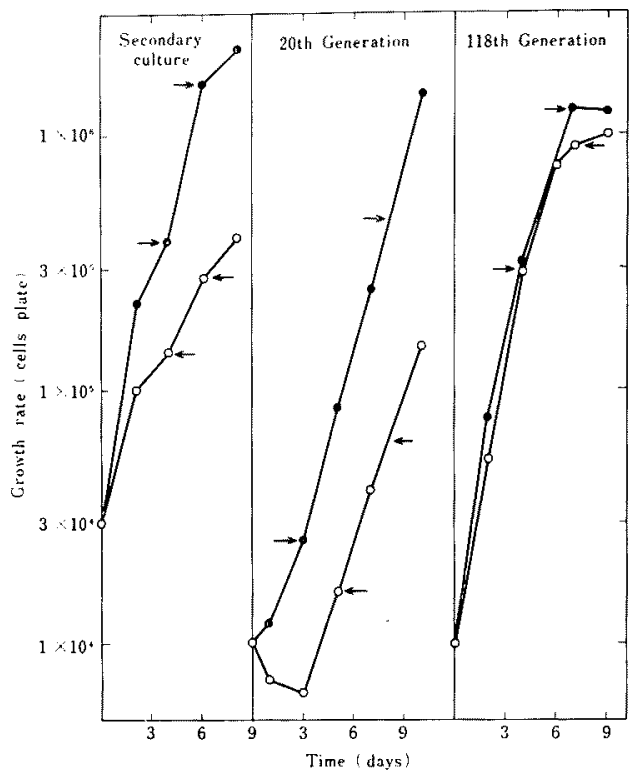

Fig. 4. Growth curves of 'A' cells inoculated to ST medium and BSA medium at different cell generations. o- $\mathrm{ST}$ medium. - - BSA medium. Arrows indicate the medium change.

secondary culture grew with a doubling time of about 50 hours at inoculum densities between $10^{3}$ and $10^{4}$ cells per plate. The cells at the 7 th cell generation or the 15th transfer grew more slowly than the cells in the secondary culture and the cells at the 11th generation or the 20th transfer could not either grow well at inoculum densities of less than 500 cells per plate. When tested at the 17 th cell generation or the 27 th transfer, however, the cells already displayed a distinct colonical growth even at an inoculum density of 300 cells per plate, while they had never grown at a cell density of less than 500 cells per plate before the 11th generation. Apparently 'A' cells in BSA medium acquired an ability to grow at lower inoculation densities at the 17 th cell generation or in 140 days.

\section{3) Change of dependency on albumin}

While the growth-promoting effect of albumin was apparent on hamster cells, albumin requirement for the cells was abated stepwise with the time spent in culture as shown previously (Fig. 2). This tendency was obviously demonstrated on the growth curves of the cells in both ST medium and BSA medium tested at different times as indicated in Fig. 4.

With secondary cultures of the cells, the growth-promoting effect of serum albumin was obvious. When tested at the 20 th cell generation or the 28 th transfer, growth curves of the cells in both media showed a similar linear increase, although the cells in ST medium had a lag phase during the initial three days. At the 20th cell generation, therefore, albumin seems to be useful to shorten the 


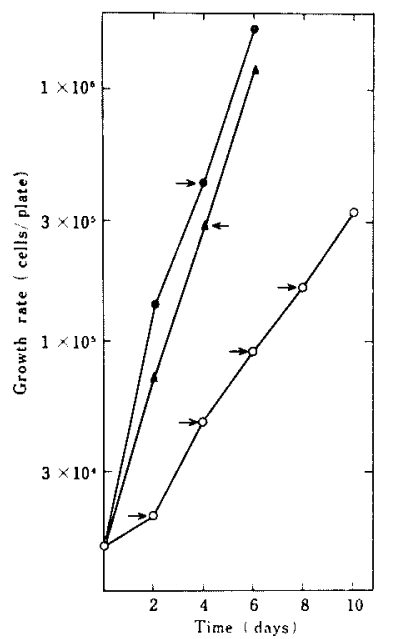

Fig. 5. Growth rate of 'A' cells in media with or without the supplement of serum. •-. BSA medium containing 10 per cent serum. 1 - BSA medium containing 2 per cent serum. ㄴ serum-free medium (Eagle's MEM plus 0.1 per cent Bacto-peptone plus low molecular substances). ${ }^{2}$ Arrows indicate the medium change.

period of lag phase appearing in ST medium. At the 118th cell generation or the 90th transfer the effect of albumin was hardly observable in both growth curves. Thus the requirement of albumin gradually disappeared with the lapse of time in culture.

\section{4) Change of dependency on serum}

Serum requirement for cells has been found to be reduced with the lapse of time in culture. ${ }^{6}$ The hamster cells could not propagate under a serum-free condition. The established lines, however, showed a progressive change concerning the serum requirement with the time spent in culture. The property changes of these cell lines were confirmed when tested at the 135 th cell generation or the 105th transfer. The patterns of the growth curves of ' $A$ ' cells in BSA medium containing either 10 per cent or 2 per cent serum were essentially similar. The cells kept in BSA medium were inoculated into serum free medium at an inoculum size of $1.5 \times 10^{4}$ cells per plate and the serum free medium was changed every two days. The harvested cells were stained with trypan blue to ensure the viable cell count. Fig. 5 shows the growth patterns of the cells at the 135 th cell generation in various media. The cells in the serum-free medium could grow with a doubling time of 55 hours, but the serial transfers were unsuccessful in this medium. The results indicate that the cells were capable of continuing cell division for several times under a serum-free condition.

\section{5) Production of tumor and re-culture}

It is known that cell lines derived from normal tissues or cells could eventually 
acquire neoplastic properties during the long term culture, as demonstrated by many investigators. ${ }^{6-11}$ Therefore, it is expected that the 'A' cell line may also acquire a neoplastic property during the long term culture. The cell cultures at the 90 th cell generation or the 70th transfer were used to examine whether they had already obtained neoplastic property. The cells were injected subcutaneously into two young hamsters. The production of tumors were ascertained at the 70th and $82 \mathrm{nd}$ days, indicating that the established cells were already tumorigenic. The cells from the tumor tissue were re-cultured in vitro and serially transferred. When the cells were cultured in BSA medium, the growth rate gradually declined. Albumin in the medium became progressively growth-inhibitory for the tumor cells.

\section{6) Morphology of the established lines}

'A' cells carried in BSA medium obtained an ability to grow at a low inoculum density at the 17 th cell generation or the 27 th transfer as described previously. The morphology of the cells between the 29th and 36th transfers is demonstrated in Figs. 6a-6h. One hundred cells were inoculated onto the plate and incubated for 12 days and the colonies formed were fixed and stained. All the cells were originally derived from the same cell population. Small epithelial cells formed various compact colonies (Figs. 6a and 6b). In the case of 'A-1' cells, the parallel orientation of fibroblastic cells which contained some polygonal cells with large cytoplasm was observed in their colonies (Figs. 6c and 6d). The colonial morphology of 'A-2' cells with a doubling time of 20 hours showed characteristic polygonal monolayers and the colonies showed a large diffuse form (Figs. 6e and 6f). On the contrary, the colonies of 'A-3' cells exhibited a typical fibroblastic morphology showing well oriented bundles of the cells (Figs. $6 \mathrm{~g}$ and $6 \mathrm{~h}$ ).

Various colonies appeared after the culture were likely to be composed of cells of different morphological types, and the existence of the mixed cell populations would indicate the persistence of a variety of cells present in the original cell population. The colonies of the cells at the 98th transfer showed a slight tendency toward piling up, which is one of the characteristics of malignant cells (Fig. 6i). The cells of confluent cultures were of polygonal or satellite shape and contained round or oval nuclei (Fig. 6j). 'A' cells had already acquired a neoplastic property at the 70 th transfer. When the cells were recultured in vitro, the cellular morphology was quite different from those of the original ' $A$ ' cells. They were small, thin and fusiform with round or oval nuclei (Fig. 7).

\section{7) Chromosomal changes of the cells}

In order to investigate the relation between the change of growth property and karyotype, chromosomal alterations were examined in the course of the development into established lines. Initial chromosomal analysis was performed with the cells at the 17 th cell generation or the 27 th transfer, where the cells just acquired 
an ability to grow at a low inoculum density. Chromosomal preparations at that generation showed a typical diploid karyotype. Therefore, the diploid cells acquired an ability to grow at a low inoculum density, before they shift to heteroploid cells. The spontaneous chromosomal alterations were not found as yet in the cells at the 31 st cell generation or the 37 th transfer. The chromosomal preparations after the following 7 serial transfers, however, demonstrated the chromosomal change to the heteroploid range with a mode of 45 and subsequently modal number of 46 .

\section{Drscussion}

Lung cells of newborn hamsters maintained in ST medium could not grow for more than 10 transfers. ${ }^{1-3}$ No established lines developed from the cultures under such a condition. Cultures of the same origin carried in BSA medium could be successively transferred and they eventually developed into established lines.

While ' $A$ ' cells were serially cultivated in the culture bottle without supplement of $\mathrm{CO}_{2}$ gas, the other cells (' $\mathrm{B}$ ' $\sim$ ' $\mathrm{E}$ ') were transferred employing the Petri dish in a $\mathrm{CO}_{2}$ incubator throughout the experiment. However, almost all the cells finally developed into established lines, although those kept in the Petri dish evolved into established lines earlier and more easily than ' $A$ ' cells. When the hamster cells developed into established lines, they always exhibited various new properties. ${ }^{3}$ The cells in the course of development into established line showed an increased growth rate in BSA medium at the 12 th cell generation as the first evidence of
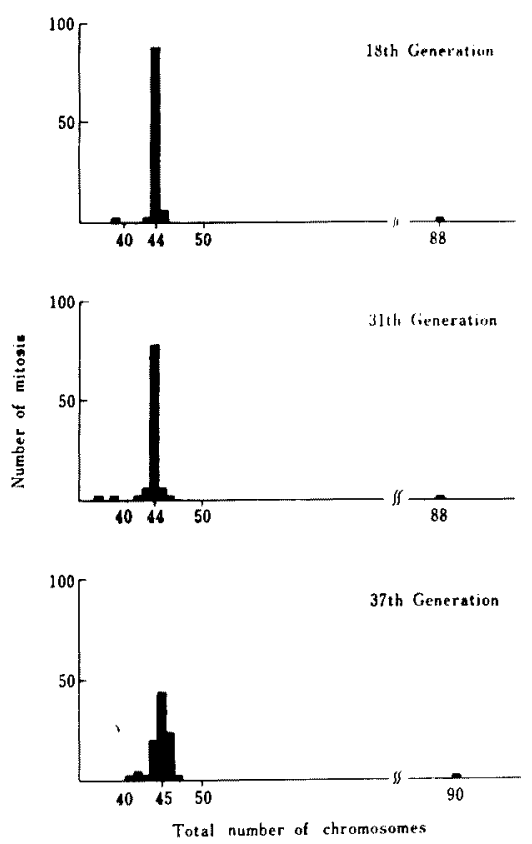

Fig. 8. Chromosomal alterations during the process of cell establishment. 
development into the established lines and then they acquired immediately an ability to sustain infinite growth in ST medium at the 14th cell generation. Consequently, the cells never died out in vitro and grew well in both BSA medium and ST medium until the experiment was finished.

One of the marked differences between freshly isolated cells and the established lines derived from them is that the latter can grow at a low inoculation density. ${ }^{5}$ After ' $A$ ' cells acquired an infinite growth potential in ST medium, they progressively obtained also an ability to grow at a low inoculation density with time spent in culture and the development of the ability was accomplished at the 17th cell generation. As the result of the cell establishment, many clones were easily formed in BSA medium but the development of the ability of colony formation in ST medium was slower and accomplished only at the 45th cell generation.

So far, it has been demonstrated by many investigators ${ }^{7-11}$ that cell lines originating from normal cell populations eventually acquire neoplastic properties during long term cultures. When 'A' cells became the established lines, they also obtained a capacity of producing tumor at the 90th cell generation and the morphology of the malignant cells was quite different from that of early established cells. Thus, the cells acquired at least two independent capacities. The neoplastic property developed far later than the capacity of producing established cell lines. Similar observation was reported previously by Todaro et al. ${ }^{12}$ with respect to the study of F-10 line. Chromosomal study of ' $A$ ' cells shows that the diploid cells were maintained until the 35th cell generation, while they had already acquired an ability to grow at a low inoculation density at the 17 th cell generation. Namely, the emergence of the neoplastic property was followed by gross chromosomal changes. In our experiments the cells carried in BSA medium acquired the ability to grow rapidly in both BSA medium and ST medium, just before the ploidy changed (Fig. 2). It may appear, therefore, that the karyotypic alteration was not essential for the establishment of cell lines.

Cultures derived from human diploid cells proliferated 5 to 11 months in vitro before they ceased to grow. ${ }^{13-15}$ Cell strains from mouse ${ }^{5,7}$ and Syrian hamster ${ }^{12.16}$ are more difficult to keep. Rothefels $e t a l .^{7}$ found that the use of $\mathrm{x}$-irradiated feeders considerably delayed the appearance and ascendancy of aneuploid cells. In the present experiment the shift of chromosomal numbers of ' $A$ ' cells emerged later at the 40th transfer, but those of the other cells emerged earlier and became apparent at near the 20th transfer. While ' $A$ ' cells were maintained in culture bottles, the other cells were sustained in Petri dishes in a $\mathrm{CO}_{2}$ incubator. All the cells on Petri dishes became established more rapidly and showed considerably less preliminary decline in their growth rate than the cells in bottle cultures. It may be suggested that the use of the culture bottle instead of the Petri dish considerably delays the appearance and ascendancy of aneuploid cells. 


\section{References}

1) Matsuya, Y. \& Yamane, I. Establishment of Syrian hamster fibroblasts in albumin fortified medium. Proc. Soc. exp. Biol. Med. (N.Y.), 1970, 134, in press.

2) Matsuya, Y. \& Yamane, I. Culture conditions necessary to sustain the serial culture of lung cells of newborn hamster in albumin fortified medium. Tohoku J. exp. Med., 1970, 101,

3) Matsuya, Y. \& Yamane, I. Serial culture of Syrian hamster fibroblasts in albumin fortified medium and their regular development into established lines. Exp. Cell Res., $1968,50,652-654$.

4) Rothfels, K.H. \& Shiminovitch, L. An air-drying technique for flattening chromosomes in mammalian cells grown in vitro. Stain Tech., 1958, 33, 73-77.

5) Todaro, G.J. \& Green, H. Quantitative studies of the growth of mouse embyro cells in culture and their development into established lines. J. Cell Biol., 1963, 17, 299-313.

6) Evans, V.J., Bryant, J.C., Kerr, H.A. \& Schilling, E.L. Chemically defined media for cultivation of long-term cell strains from four mammalian species. Exp. Cell Res., 1964, 36, 439-474.

7) Rothfels, K.H., Kupelwieser, E.B. \& Parker, R.C. Effect of X-irradiated feeder layers on mitotic activity and development of aneuploid in mouse embyro cells in vitro. Fifth Canadian Cancer Conference (Academic Press), 1963, 5, 191-223.

8) Evans, V.J., Parker, G.A. \& Dunn, T.B. Neoplastic transformations in $\mathrm{C}_{3} \mathrm{H}$ mouse embyronic tissue in vitro determined by intraocular growth. I. Cells from chemically defined medium with and without serum supplement. $J$, nat. Cancer Inst., 1964, 32, 89-121.

9) Yamane, I. \& Tsuda, T. Malignant transformation and chromosome change of two cell lines from Syrian hamster embyronic tissue in vitro. Tohoku J. exp. Med., 1966, 88, 171-180.

10) Stoker, M. \& Macpherson, I. Syrian hamster fibroblast cell line BHK 21 and its derivatives. Nature, 1964, 203, 1355-1357.

11) Diamond, L. Two spontaneously transformed cell lines derived from the same hamster embyro culture. Int. J. Cancer, 1967, 2, 143-152.

12) Todaro, G.J., Nilausen, K. \& Green, H. Growth properties of polyoma virus-induced hamster tumor cells. Cancer Res., 1963, 23, 825-832.

13) Hayflick, L. \& Moorhead, P.S. The serial cultivation of human diploid cell strains. Exp. Cell Res., 1961, 25, 585-621.

14) Hayflick, L. The limited in vitro life time of human diploid cell strains. Exp. Cell Res., 1965, 37, 614-636.

15) Todaro, G.J., Wolman, S.R. \& Green, H. Rapid transformation of human fibroblasts with low growth potential into established cell lines by SV40. J. Cell comp. Physiol., $1963,62,257-265$.

16) Defendi, V. \& Lehman, J.M. Transformation of hamster embryo cells in vitro by polyoma virus: morphological, karyological, immunological and transplantation chara. cteristics. J. Cell comp. Physiol., 1965, 66, 351-410. 
Fig. 6a. 'A' cells were continuously cultured from the primry culture and formed the colonies at the 17 th generation. $\times 1.7$.

Fig. 6b. The enlargement of a portion of Fig. $6 \mathrm{a} . \quad \times 44$.

Fig. 6c. A-1 cells were derived from a massive culture of ' $A$ ' cells at 12th generation where fibroblastic cells were quite predominant. The colonies were formed at the 17th generation. $\times 1.7$

Fig. 6d. The enlargement of a portion of Fig. $6 \mathrm{c} . \quad \times 44$.

Fig. 6e. A-2 cells were isolated from a colony of ' $A$ ' cells at the 20 th generation. $\times 1.7$.

Fig. 6f. The enlargement of a portion of Fig. 6e. $\times 44$. 

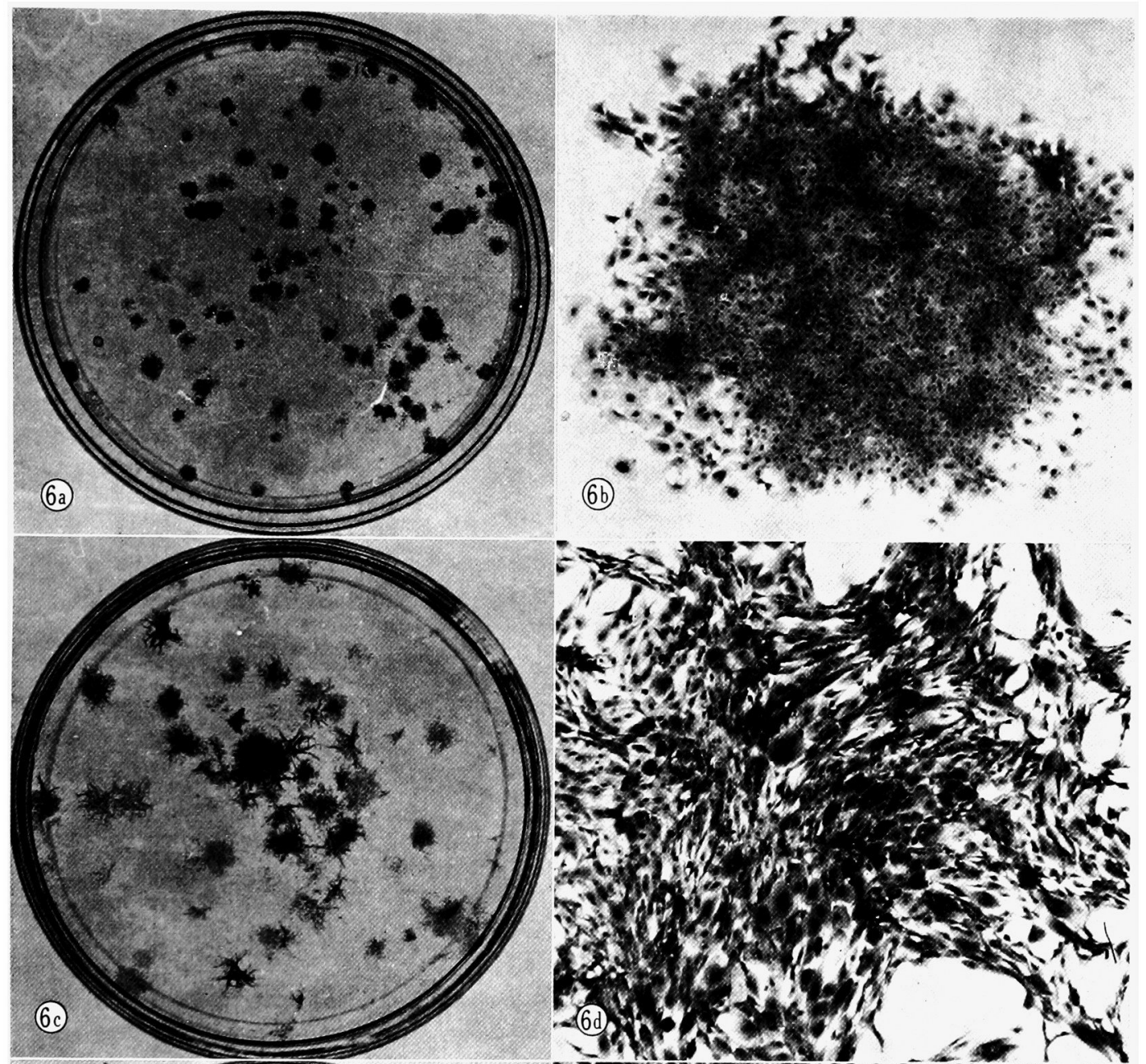

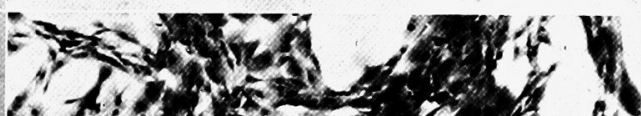

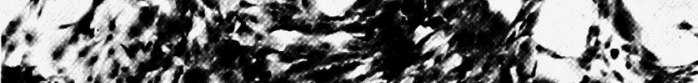

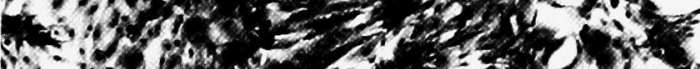

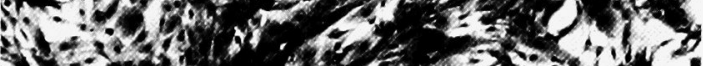
$1-2,20021$ ?

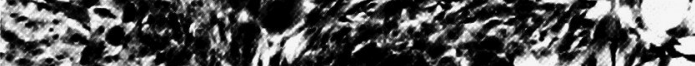

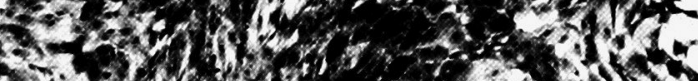

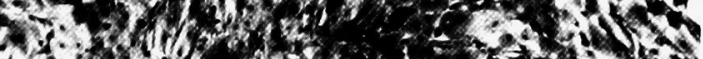

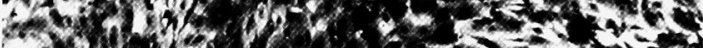

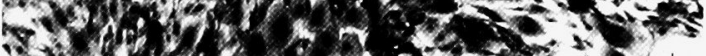

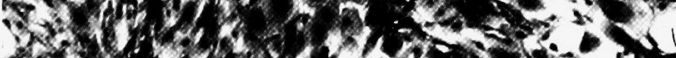

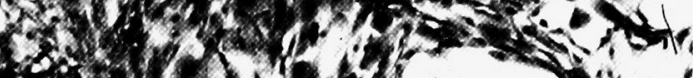

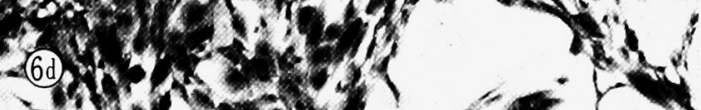

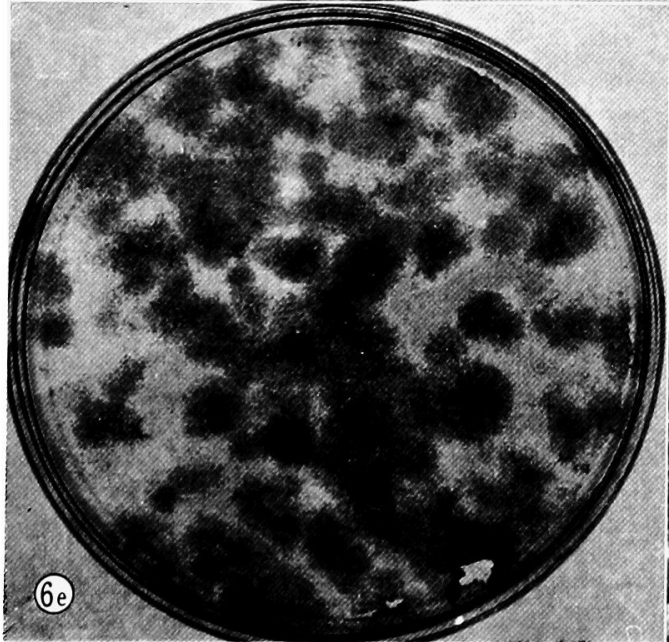

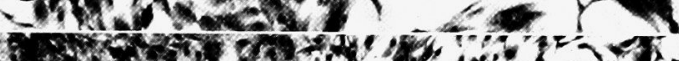

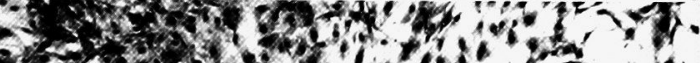

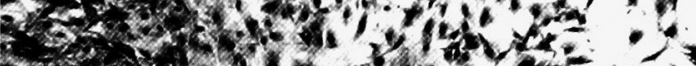

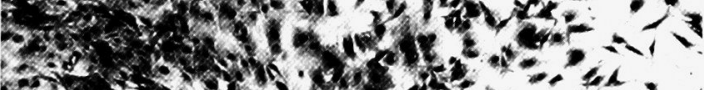

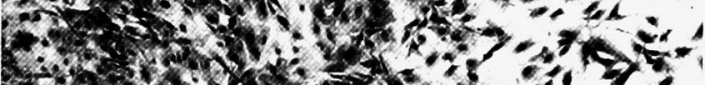

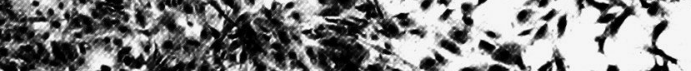

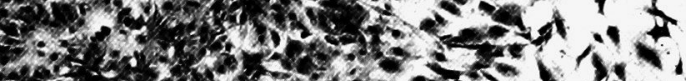

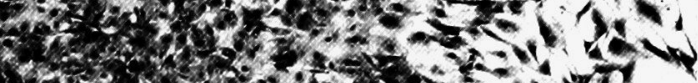

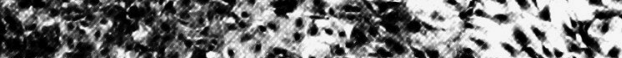
2. 10 .

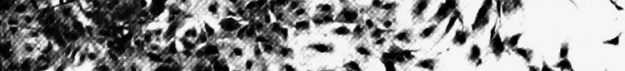

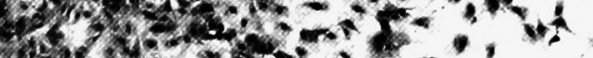

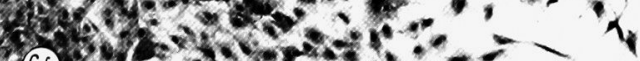

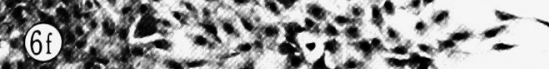


Fig. 6g. A-3 cells were isolated from a colony of ' $A$ ' cells at the 22 nd generation. $\times 1.7$. Fig. 6h. The enlargement of a portion of Fig. $6 \mathrm{~g} . \quad \times 44$.

Fig. 6i. Colony of ' $A$ ' cells at the 130th generation. $\times 44$.

Fig. 6j. Monolyaer of ' $A$ ' cells at the 130th generation. $\times 220$.

Fig. 7. A portion of a colony originated from tumor cells. $\times 220$. 

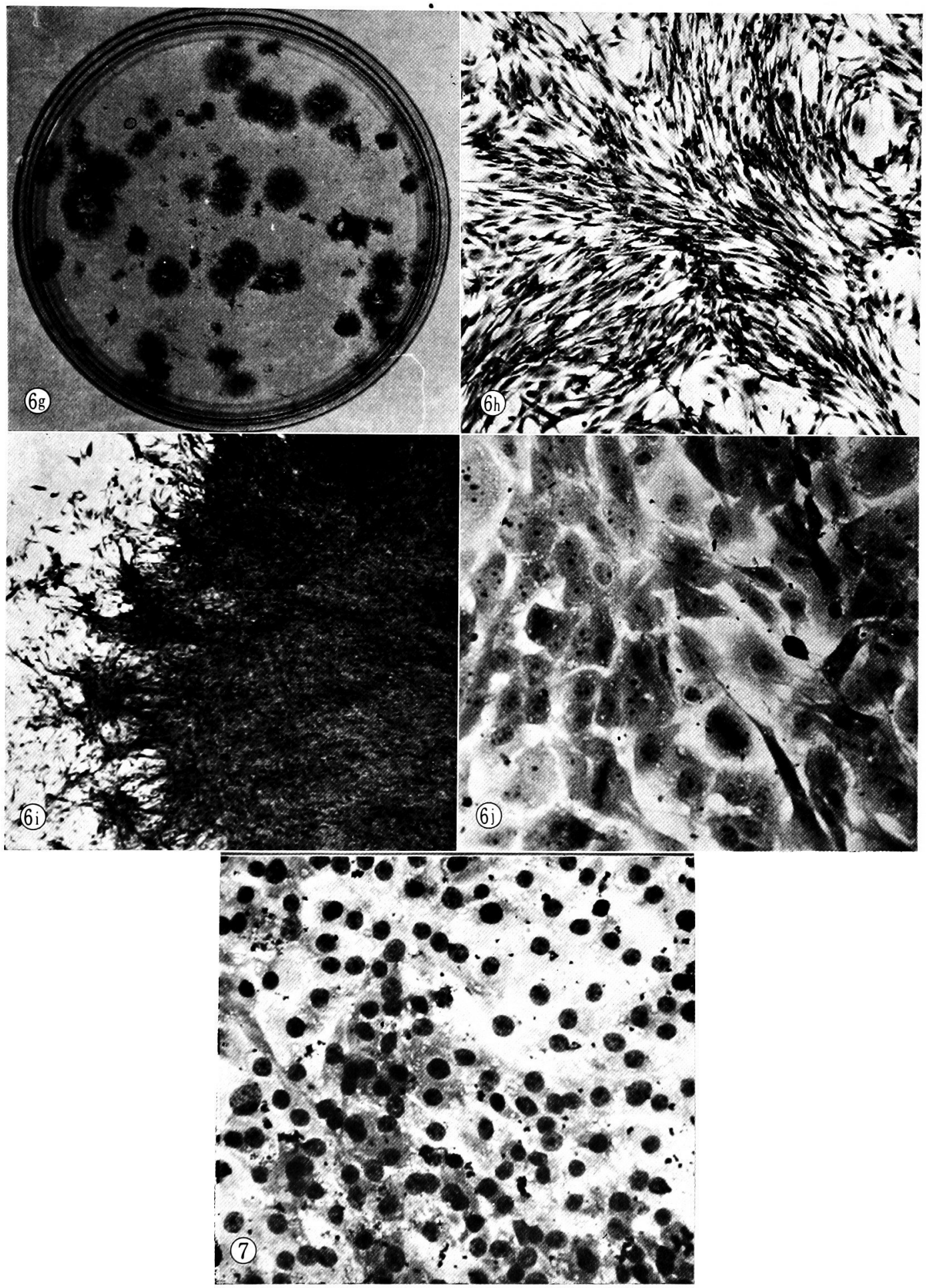